Supplemental Material

\title{
Kekulé Lattice in Graphdiyne: Coexistence of Phononic and Electronic Second-Order Topological Insulator
}

\author{
Haimen $\mathrm{Mu}^{1}$, Bing $\mathrm{Liu}^{1}$, Tianyi $\mathrm{Hu}^{1}$, and Zhengfei Wang ${ }^{1, *}$ \\ ${ }^{1}$ Hefei National Laboratory for Physical Sciences at the Microscale, CAS Key \\ Laboratory of Strongly-Coupled Quantum Matter Physics, Department of Physics, \\ University of Science and Technology of China, Hefei, Anhui 230026, China
}

*Correspondence to: zfwang15@ustc.edu.cn

\section{Density-Functional Tight-Binding Calculations}

The electronic structures of graphdiyne are calculated by density-functional tightbinding (DFTB) approach in DFTB+ package ${ }^{1}$. The Slater-Koster pbc-parameters are used to describe the coupling between carbon atoms with one $s$ and three $p$ orbitals ${ }^{2}$. The $21 \times 21 \times 1$ Monkhorst-Pack $k$-point mesh is used in the self-consistent calculations. During the structure optimization, all atoms are relaxed until force less than $10^{-9}$ Hartree/Bohr and stress less than $10^{-10}$ Hartree/Bohr ${ }^{3}$. The optimized lattice constant is 17.996 Bohr. The electron TB Hamiltonian $(H)$ and overlap matrix $(S)$ are obtained from the DFTB+.

In order to check the effect of edge structural relaxation for gapped topological edge state and in-gap topological corner state shown in Fig. 2 in main text, we further relax 
a narrow graphdiyne nanoribbon and small graphdiyne cluster to directly extract force constants by including relaxation induced Hamiltonian variation. The corresponding out-of-plane and in-plane phonon bands, and spatial distribution of corner modes are shown in Fig. S1. Although the edge bands are reshaped by structural relaxation, one can see that the gapped edge state and in-gap corner state are still reserved, which are originated from the nontrivial bulk topology of graphdiyne.

For different edge configurations, the out-of-plane and in-plane phonon bands of graphdiyne nanoribbon and discrete phonon levels of hexagonal graphdiyne cluster are shown in Fig. S2. One can see that there always exists six topological corner states, but the energies can be either inside or outside the bulk gap. The electronic structures of graphdiyne are also shown in Fig. S4, exhibiting the gapped topological edge states (Fig. S4(b)) and six in-gap topological corner states (Fig. S4(c)) in the bulk gap (Fig. S4(a)). The spatial distribution of these corner states is shown in Fig. 4(b) in main text, which are localized at six corner regions. The EPC between electron states and in-plane phonon modes are shown in Fig. S6(a). Different to the out-of-plane phonon modes, there is no enhanced EPC for the electron and phonon localized at the same corner (white rectangular region), because all phononic corner states have odd mirror symmetry (Fig. S6(b)-(g)).

\section{Topological Index of Quadrupole Moment}

In a $2 \mathrm{D}$ system with both inversion and time-reversal symmetries, the dipole moment 
can be calculated by checking the parities at time-reversal-invariant-momenta as ${ }^{3}$

$$
\begin{aligned}
& p_{i}=\frac{1}{2}\left(\sum_{n} 2 p_{i}^{n}\right. \text { module 2) } \\
& p_{i}^{n}=\frac{1}{2} q_{i}^{n} \\
& (-1)^{q_{i}^{n}}=\eta^{n}(\mathrm{M}) / \eta^{n}(\Gamma)
\end{aligned}
$$

where $i=1$ or 2 is the direction of reciprocal lattice vector, $\eta^{n}(k)$ is the parity of $n$th subband at $k$-point below bulk gap, and $q_{i}^{n}$ equals either 0 or 1 . The quadrupole moment can be obtained from the dipole moment as

$$
q_{i j}=\frac{1}{2}\left(\sum_{n} 2 p_{i}^{n} p_{j}^{n} \text { module } 2\right)
$$

This definition of quadrupole moment is valid only if the dipole moment vanishes. Therefore, $q_{i j}=1 / 2$ (or 0 ) if the number of subbands with opposite parity between $\Gamma$ and $\mathrm{M}$ below the bulk gap is $2,6,10 \ldots$ (or $4,8,12 \ldots$ ), otherwise, it has no definition. The parities of graphdiyne subbands obtained from DFTB calculations are listed in Table I, showing the nontrivial topology for both out-of-plane and in-plane modes.

\section{Graphdiyne vs. Kekulé Lattice}

In graphdiyne, the phonon hopping between two vertex carbons can be regarded as a renormalized direct hopping as follows. Considering a simplified phonon Hamiltonian of graphdiyne at six carbons, as shown in Fig. S8, where $\chi_{1-6}^{x, y, z}$ is the component of phonon mode at different carbons along different directions, and $t_{1,2,3}^{x, y, z}$ is the force constant for out-of-plane $(z)$ and in-plane $(x, y)$ hopping. 
For the out-of-plane modes with nearest-neighboring hopping, the phonon equation can be written as

$$
\left(\begin{array}{cccccc}
\varepsilon & t_{1}^{z} & 0 & 0 & 0 & 0 \\
t_{1}^{z} & \varepsilon & t_{2}^{z} & 0 & 0 & 0 \\
0 & t_{2}^{z} & \varepsilon & t_{3}^{z} & 0 & 0 \\
0 & 0 & t_{3}^{z} & \varepsilon & t_{2}^{z} & 0 \\
0 & 0 & 0 & t_{2}^{z} & \varepsilon & t_{1}^{z} \\
0 & 0 & 0 & 0 & t_{1}^{z} & \varepsilon
\end{array}\right)\left(\begin{array}{c}
\chi_{1} \\
\chi_{2} \\
\chi_{3} \\
\chi_{4} \\
\chi_{5} \\
\chi_{6}
\end{array}\right)=m \omega^{2}\left(\begin{array}{c}
\chi_{1} \\
\chi_{2} \\
\chi_{3} \\
\chi_{4} \\
\chi_{5} \\
\chi_{6}
\end{array}\right)
$$

Solving this equation, if the on-site term $\varepsilon: m \omega^{2}$, one can obtain the following simple relation $\chi_{1}^{z}=\frac{t_{1}^{z 2} t_{3}^{z}}{t_{2}^{z 2}} \chi_{6}^{z}$. Therefore, the effective out-of-plane hopping between two vertex carbons is $\frac{t_{1}^{z 2} t_{3}^{z}}{t_{2}^{z 2}}$.

Similarly, for the in-plane modes with nearest-neighboring hopping, the phonon equation can be written as

$$
\left(\begin{array}{cccccccccccc}
\varepsilon & 0 & t_{1}^{x} & 0 & 0 & 0 & 0 & 0 & 0 & 0 & 0 & 0 \\
0 & \varepsilon & 0 & t_{1}^{y} & 0 & 0 & 0 & 0 & 0 & 0 & 0 & 0 \\
t_{1}^{x} & 0 & \varepsilon & 0 & t_{2}^{x} & 0 & 0 & 0 & 0 & 0 & 0 & 0 \\
0 & t_{1}^{y} & 0 & \varepsilon & 0 & t_{2}^{y} & 0 & 0 & 0 & 0 & 0 & 0 \\
0 & 0 & t_{2}^{x} & 0 & \varepsilon & 0 & t_{3}^{x} & 0 & 0 & 0 & 0 & 0 \\
0 & 0 & 0 & t_{2}^{y} & 0 & \varepsilon & 0 & t_{3}^{y} & 0 & 0 & 0 & 0 \\
0 & 0 & 0 & 0 & t_{3}^{x} & 0 & \varepsilon & 0 & t_{2}^{x} & 0 & 0 & 0 \\
0 & 0 & 0 & 0 & 0 & t_{3}^{y} & 0 & \varepsilon & 0 & t_{2}^{y} & 0 & 0 \\
0 & 0 & 0 & 0 & 0 & 0 & t_{2}^{x} & 0 & \varepsilon & 0 & t_{1}^{x} & 0 \\
0 & 0 & 0 & 0 & 0 & 0 & 0 & t_{2}^{y} & 0 & \varepsilon & 0 & t_{1}^{y} \\
0 & 0 & 0 & 0 & 0 & 0 & 0 & 0 & t_{1}^{x} & 0 & \varepsilon & 0 \\
0 & 0 & 0 & 0 & 0 & 0 & 0 & 0 & 0 & t_{1}^{y} & 0 & \varepsilon
\end{array}\right)\left(\begin{array}{c}
\chi_{1}^{x} \\
\chi_{1}^{y} \\
\chi_{2}^{x} \\
\chi_{2}^{y} \\
\chi_{3}^{x} \\
\chi_{3}^{y} \\
\chi_{4}^{x} \\
\chi_{4}^{y} \\
\chi_{5}^{x} \\
\chi_{5}^{y} \\
\chi_{6}^{x} \\
\chi_{6}^{y}
\end{array}\right)=m \omega^{2}\left(\begin{array}{c}
\chi_{1}^{x} \\
\chi_{1}^{y} \\
\chi_{2}^{x} \\
\chi_{2}^{y} \\
\chi_{3}^{x} \\
\chi_{3}^{y} \\
\chi_{4}^{x} \\
\chi_{4}^{y} \\
\chi_{5}^{x} \\
\chi_{5}^{y} \\
\chi_{6}^{x} \\
\chi_{6}^{y}
\end{array}\right)
$$

Solving this equation, if the on-site term $\varepsilon: m \omega^{2}$, one can obtain the following 
simple relation $\chi_{1}^{x}=\frac{t_{1}^{x 2} t_{3}^{x}}{t_{2}^{x 2}} \chi_{6}^{x}$ and $\chi_{1}^{y}=\frac{t_{1}^{y 2} t_{3}^{y}}{t_{2}^{y 2}} \chi_{6}^{y}$. Therefore, the effective in-plane hopping between two vertex carbons is $\frac{t_{1}^{x 2} t_{3}^{x}}{t_{2}^{x 2}}$ and $\frac{t_{1}^{y 2} t_{3}^{y}}{t_{2}^{y 2}}$. Consequently, $t_{\text {inter }}=\frac{t_{1}^{2} t_{3}}{t_{2}^{2}}$ can be used to describe both out-of-plane and in-plane hopping between carbons in different benzene rings, making graphdiyne equivalent to the Kekulé Lattice.

\section{Two-Atom Model for Electron-Phonon Coupling}

In a two-atom model with orthogonal $p_{z}$ orbital (neglecting the overlap matrix), as shown in Fig. S9, the EPC in Eq. (1) in main text can be simplified to

$$
\Xi_{v}^{n}=\sum_{\alpha} \chi_{v, i}^{\alpha}\left\langle\psi_{n}\left|\frac{\partial H}{\partial r_{i}^{\alpha}}\right| \psi_{n}\right\rangle
$$

The electron Hamiltonian at equilibrium configuration can be written as

$$
H=\left(\begin{array}{cc}
0 & \gamma_{0} \\
\gamma_{0} & 0
\end{array}\right)
$$

The corresponding electron eigenstate is

$$
\left|\psi_{1}\right\rangle=\frac{1}{\sqrt{2}}\left(\begin{array}{l}
1 \\
1
\end{array}\right),\left|\psi_{2}\right\rangle=\frac{1}{\sqrt{2}}\left(\begin{array}{c}
1 \\
-1
\end{array}\right)
$$

For the out-of-plane phonon mode (Fig. S9(a)), it can be written as

$$
\text { even mirror symmetry: } P_{1}=\left(\begin{array}{c}
\chi_{z} \\
\chi_{z}
\end{array}\right) \text {, odd mirror symmetry: } P_{2}=\left(\begin{array}{c}
\chi_{z} \\
-\chi_{z}
\end{array}\right)
$$

The out-of-plane phonon mode induced variation of electron Hamiltonian will be 


$$
\frac{\partial H}{\partial r_{z}^{1}} \approx \frac{\Delta H}{\Delta r_{z}^{1}}=\left(\begin{array}{cc}
0 & \frac{\gamma_{1}-\gamma_{0}}{\Delta r_{z}^{1}} \\
\frac{\gamma_{1}-\gamma_{0}}{\Delta r_{z}^{1}} & 0
\end{array}\right)=\left(\begin{array}{cc}
0 & \frac{\gamma_{1}-\gamma_{0}}{\Delta r_{z}^{2}} \\
\frac{\gamma_{1}-\gamma_{0}}{\Delta r_{z}^{2}} & 0
\end{array}\right)=\frac{\Delta H}{\Delta r_{z}^{2}} \approx \frac{\partial H}{\partial r_{z}^{2}}
$$

where the tiny out-of-plane displacement $\Delta r_{z}^{1}=\Delta r_{z}^{2}$. Therefore, the EPC between electron state $\left|\psi_{1,2}\right\rangle$ and phonon mode $P_{1}$ with even mirror symmetry are

$$
\begin{aligned}
& \Xi_{1}^{1}=\left\langle\psi_{1}\left|\frac{\partial H}{\partial r_{z}^{1}}\right| \psi_{1}\right\rangle \chi_{z}+\left\langle\psi_{1}\left|\frac{\partial H}{\partial r_{z}^{2}}\right| \psi_{1}\right\rangle \chi_{z}=\frac{2\left(\gamma_{1}-\gamma_{0}\right)}{\Delta r_{z}^{1}} \chi_{z} \\
& \Xi_{1}^{2}=\left\langle\psi_{2}\left|\frac{\partial H}{\partial r_{z}^{1}}\right| \psi_{2}\right\rangle \chi_{z}+\left\langle\psi_{2}\left|\frac{\partial H}{\partial r_{z}^{2}}\right| \psi_{2}\right\rangle \chi_{z}=\frac{-2\left(\gamma_{1}-\gamma_{0}\right)}{\Delta r_{z}^{1}} \chi_{z}
\end{aligned}
$$

The EPC between electron state $\left|\psi_{1,2}\right\rangle$ and phonon mode $P_{2}$ with odd mirror symmetry are

$$
\begin{aligned}
& \Xi_{2}^{1}=\left\langle\psi_{1}\left|\frac{\partial H}{\partial r_{z}^{1}}\right| \psi_{1}\right\rangle \chi_{z}+\left\langle\psi_{1}\left|\frac{\partial H}{\partial r_{z}^{2}}\right| \psi_{1}\right\rangle\left(-\chi_{z}\right)=0 \\
& \Xi_{2}^{2}=\left\langle\psi_{2}\left|\frac{\partial H}{\partial r_{z}^{1}}\right| \psi_{2}\right\rangle \chi_{z}+\left\langle\psi_{2}\left|\frac{\partial H}{\partial r_{z}^{2}}\right| \psi_{2}\right\rangle\left(-\chi_{z}\right)=0
\end{aligned}
$$

Therefore, the EPC is non-zero for out-of-plane phonon modes with even mirror symmetry only, which is irrelevant to the symmetry of electron eigenstates.

For the in-plane mode (Fig. S9(b)), it can be written as

$$
\text { even mirror symmetry: } P_{1}=\left(\begin{array}{c}
\chi_{y} \\
-\chi_{y}
\end{array}\right) \text {, odd mirror symmetry: } P_{1}=\left(\begin{array}{c}
\chi_{y} \\
\chi_{y}
\end{array}\right)
$$

The in-plane phonon mode induced variation of electron Hamiltonian is

$$
\frac{\partial H}{\partial r_{y}^{1}} \approx \frac{\Delta H}{\Delta r_{y}^{1}}=\left(\begin{array}{cc}
0 & \frac{\gamma_{2}-\gamma_{0}}{\Delta r_{y}^{1}} \\
\frac{\gamma_{2}-\gamma_{0}}{\Delta r_{y}^{1}} & 0
\end{array}\right), \frac{\partial H}{\partial r_{y}^{2}} \approx \frac{\Delta H}{\Delta r_{y}^{2}}=\left(\begin{array}{cc}
0 & \frac{\gamma_{3}-\gamma_{0}}{\Delta r_{y}^{2}} \\
\frac{\gamma_{3}-\gamma_{0}}{\Delta r_{y}^{2}} & 0
\end{array}\right)
$$

Considering the exponential decay of hopping parameter, one can get 


$$
\begin{aligned}
& \gamma_{2}=\gamma_{0} \exp \left(1-\frac{d_{0}-\Delta r_{y}^{1}}{d_{0}}\right) \approx \gamma_{0}\left(1+\frac{\Delta r_{y}^{1}}{d_{0}}\right) \\
& \gamma_{3}=\gamma_{0} \exp \left(1-\frac{d_{0}+\Delta r_{y}^{2}}{d_{0}}\right) \approx \gamma_{0}\left(1-\frac{\Delta r_{y}^{2}}{d_{0}}\right)
\end{aligned}
$$

where $d_{0}$ is the distance between two atoms at equilibrium configuration. The EPC between electron state $\left|\psi_{1,2}\right\rangle$ and phonon mode $P_{1}$ with even mirror symmetry are

$$
\begin{aligned}
& \Xi_{1}^{1}=\left\langle\psi_{1}\left|\frac{\partial H}{\partial r_{y}^{1}}\right| \psi_{1}\right\rangle \chi_{y}+\left\langle\psi_{1}\left|\frac{\partial H}{\partial r_{y}^{2}}\right| \psi_{1}\right\rangle\left(-\chi_{y}\right)=\left(\frac{\gamma_{2}-\gamma_{0}}{\Delta r_{y}^{1}}-\frac{\gamma_{3}-\gamma_{0}}{\Delta r_{y}^{2}}\right) \chi_{y} \approx \frac{2 \gamma_{0}}{d_{0}} \chi_{y} \\
& \Xi_{1}^{2}=\left\langle\psi_{2}\left|\frac{\partial H}{\partial r_{y}^{1}}\right| \psi_{2}\right\rangle \chi_{y}+\left\langle\psi_{2}\left|\frac{\partial H}{\partial r_{y}^{2}}\right| \psi_{2}\right\rangle\left(-\chi_{y}\right)=-\left(\frac{\gamma_{2}-\gamma_{0}}{\Delta r_{y}^{1}}-\frac{\gamma_{3}-\gamma_{0}}{\Delta r_{y}^{2}}\right) \chi_{y} \approx-\frac{2 \gamma_{0}}{d_{0}} \chi_{y}
\end{aligned}
$$

The EPC between electron state $\left|\psi_{1,2}\right\rangle$ and phonon mode $P_{2}$ with odd mirror symmetry is

$$
\begin{aligned}
& \Xi_{2}^{1}=\left\langle\psi_{1}\left|\frac{\partial H}{\partial r_{y}^{1}}\right| \psi_{1}\right\rangle \chi_{y}+\left\langle\psi_{1}\left|\frac{\partial H}{\partial r_{y}^{2}}\right| \psi_{1}\right\rangle \chi_{y}=\left(\frac{\gamma_{2}-\gamma_{0}}{\Delta r_{y}^{1}}+\frac{\gamma_{3}-\gamma_{0}}{\Delta r_{y}^{2}}\right) \chi_{y} \approx 0 \\
& \Xi_{2}^{2}=\left\langle\psi_{2}\left|\frac{\partial H}{\partial r_{y}^{1}}\right| \psi_{2}\right\rangle \chi_{y}+\left\langle\psi_{2}\left|\frac{\partial H}{\partial r_{y}^{2}}\right| \psi_{2}\right\rangle \chi_{y}=-\left(\frac{\gamma_{2}-\gamma_{0}}{\Delta r_{y}^{1}}+\frac{\gamma_{3}-\gamma_{0}}{\Delta r_{y}^{2}}\right) \chi_{y} \approx 0
\end{aligned}
$$

Therefore, the EPC is non-zero for in-plane phonon modes with even mirror symmetry only, which is irrelevant to the symmetry of electron eigenstates.

\section{Reference}

1. Hourahine, B. et al., DFTB+, a software package for efficient approximate density functional theory based atomistic simulations. J. Chem. Phys. 2020, 152, 124101.

2. Kohler, C.; Frauenheim, T. Molecular dynamics simulations of $\mathrm{CF}_{\mathrm{x}}(\mathrm{x}=2,3)$ molecules at $\mathrm{Si}_{3} \mathrm{~N}_{4}$ and $\mathrm{SiO}_{2}$ surfaces. Sur. Sci. 2006, 600, 453.

3. Liu, F.; Deng, H. Y.; Wakabayashi, K. Helical topological edge states in a quadrupole phase. Phys. Rev. Lett. 2019, 122, 086804. 


\begin{tabular}{|c|c|c|c|c|c|c|c|c|c|c|c|c|c|c|c|c|c|c|c|c|c|c|}
\hline TRIM & \multicolumn{10}{|c|}{ In-plane mode parity } \\
\hline$\Gamma$ & - & - & + & - & - & - & + & + & + & + & + & - & - & - & - & + & + & + & - & - & + & - \\
\hline $\mathbf{M}$ & + & - & + & + & - & - & + & + & - & - & - & + & - & + & + & - & + & - & + & - & + & - \\
\hline
\end{tabular}

\begin{tabular}{|c|c|c|c|c|c|c|c|c|c|c|c|c|c|c|c|c|c|c|}
\hline TRIM & \multicolumn{10}{|c|}{ Out-of-plane mode parity } \\
\hline$\Gamma$ & - & - & - & + & + & - & + & - & - & + & + & - & + & + & + & - & - & + \\
\hline $\mathbf{M}$ & - & + & + & - & + & + & - & - & + & - & - & + & - & + & + & - & - & + \\
\hline
\end{tabular}

Table-I. Parities of subbands at $\Gamma$ and $M$ points for in-plane and out-of-plane modes of graphdiyne, where label $+/-$ denotes even/odd parity. At each TRIM, from left to right, the parities are sorted from low to high phonon frequency. The parities below the bulk gap are highlighted by grey color. 

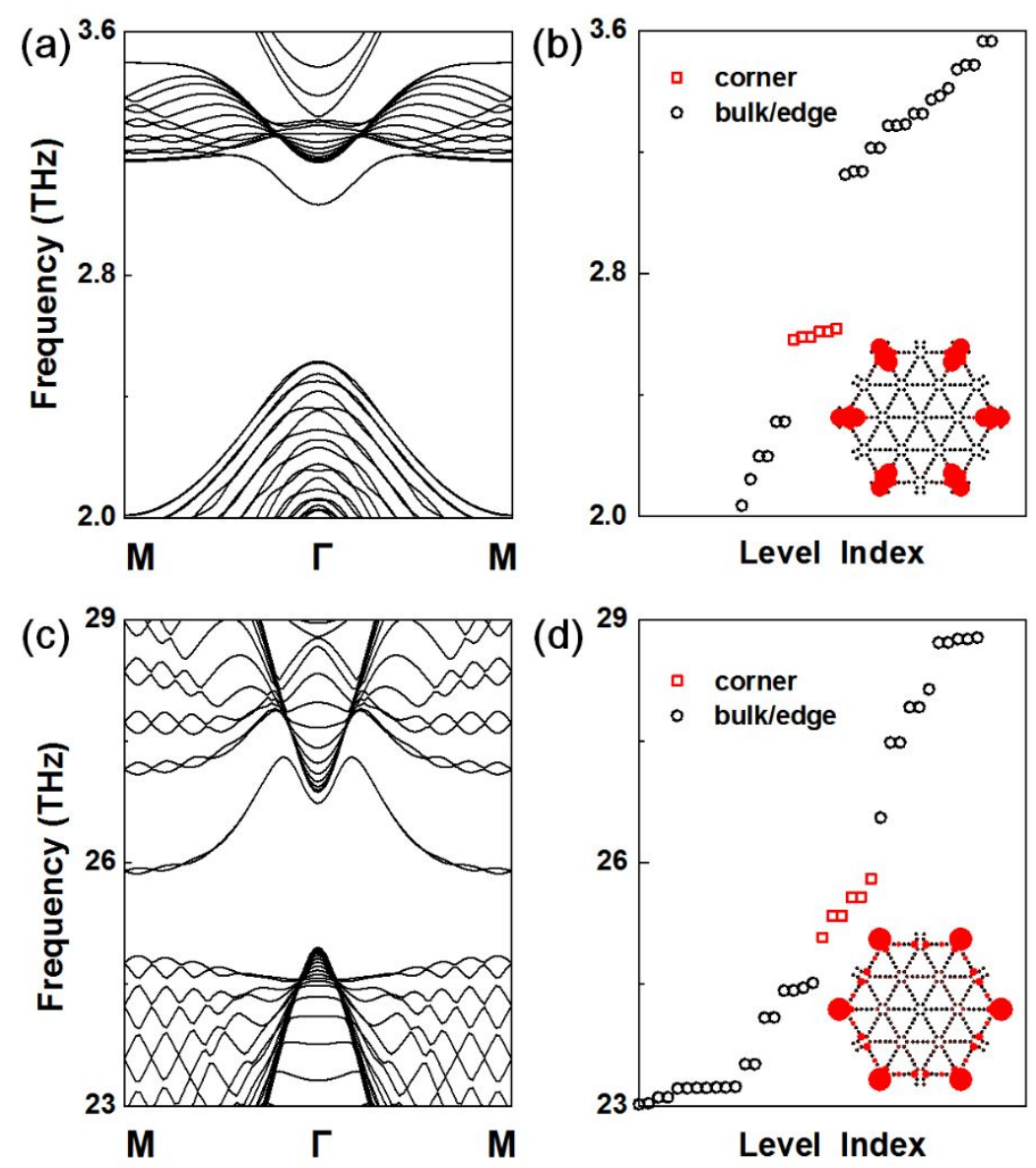

Figure S1. Phononic SOTI in graphdiyne by including edge structural relaxation. (a) Out-of-plane phonon bands of graphdiyne nanoribbon (13 unit-cell width) calculated from the relaxed structure with the same edge termination as that in Fig. 2(b). (b) Discrete out-of-plane phonon levels of hexagonal graphdiyne cluster calculated from the relaxed structure with spatial distribution of six corner states shown in inset. (c)-(d) are the same to (a)-(b), but for in-plane modes of graphdiyne. 

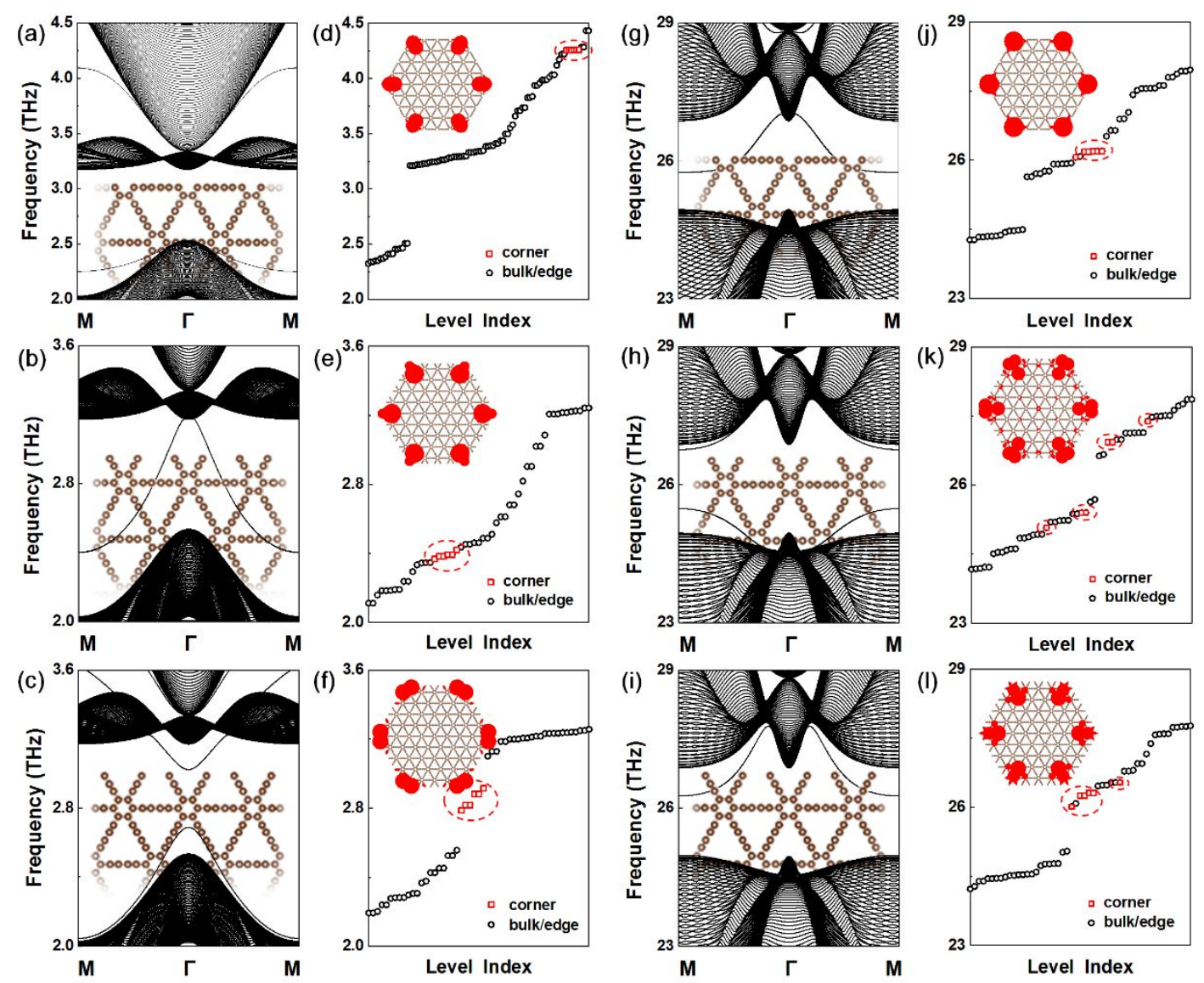

Figure S2. (a)-(c) Out-of-plane phonon bands of graphdiyne nanoribbon with different edge terminations shown in inset. (d)-(f) The corresponding discrete out-of-plane phonon levels of hexagonal graphdiyne cluster for (a)-(c) with spatial distribution of six corner states shown in inset. (g)-(l) are the same to (a)-(f), but for in-plane modes of graphdiyne. The six robust topological corner states are highlighted in the red-dashed circle, which can be either inside or outside the bulk gap in (d)-(f) and (j)-(1). 


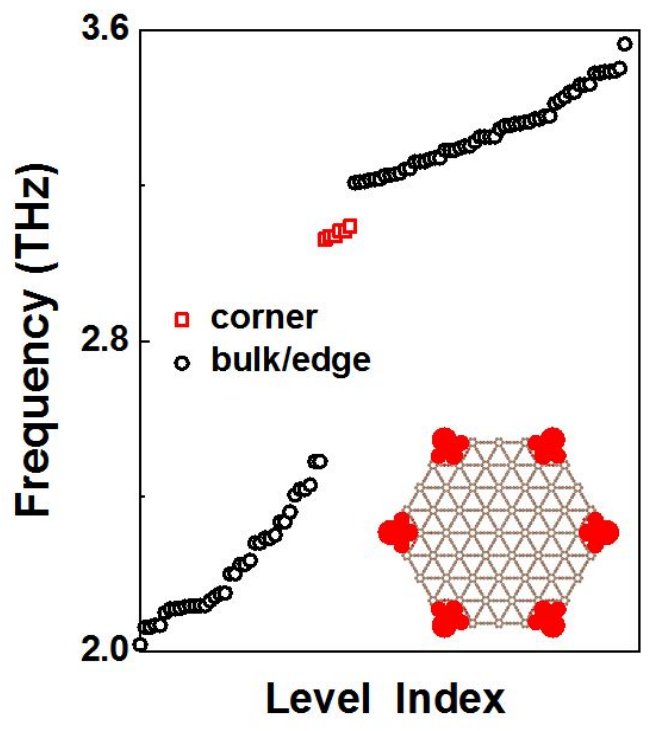

Figure S3. Discrete out-of-plane phonon levels of hexagonal graphdiyne cluster with local corner potential added to outmost corner atoms without the largest vibration amplitude. The inset is the corresponding spatial distribution of six corner states, which is slightly different to that shown in Fig. 3(b) inset without local corner potential. 

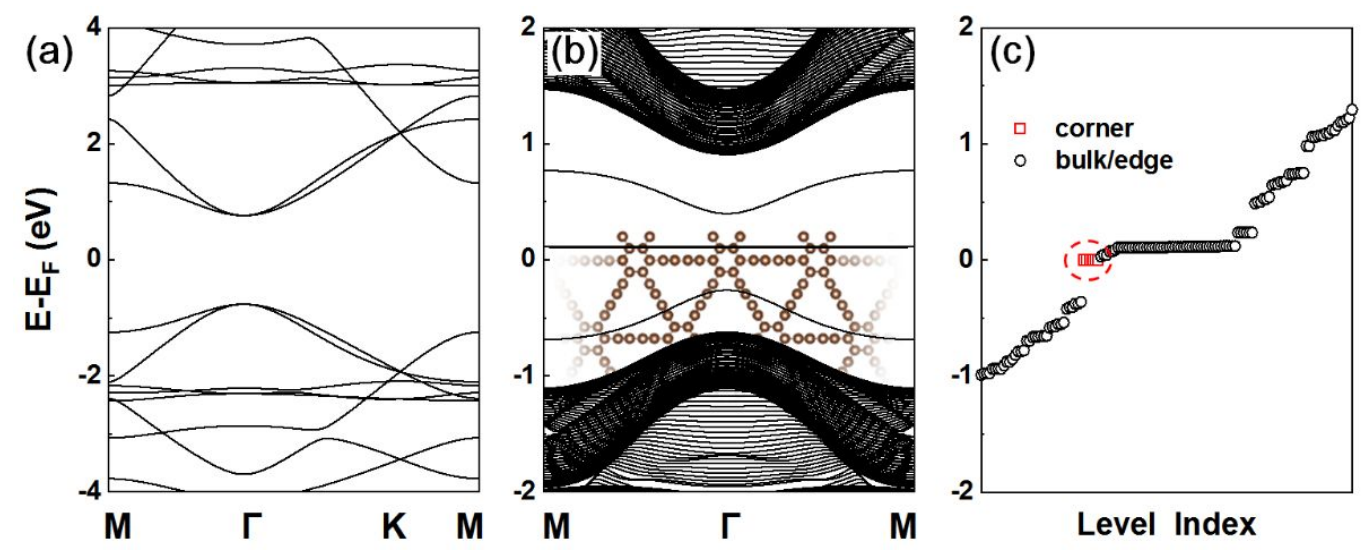

Figure S4. (a) Electron bulk bands of graphdiyne. (b) Electron bands of graphdiyne nanoribbon with edge termination shown in inset. (c) Discrete electron levels of hexagonal graphdiyne cluster with six corner states highlighted in the red-dashed circle. 

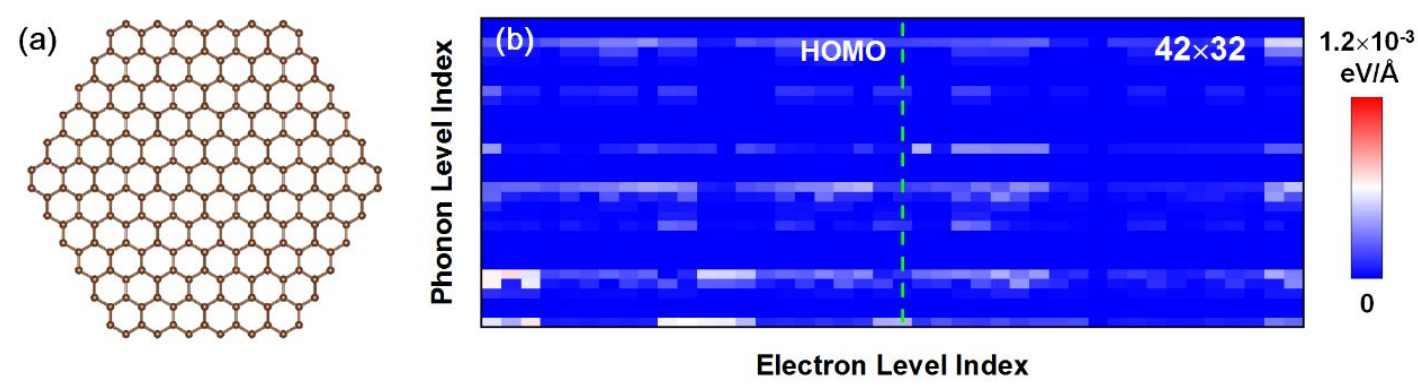

Figure S5. The EPC in graphene. (a) The hexagonal cluster of the graphene with zigzag edge termination. (b) The EPC between 42 electron states around Fermi-level and 32 out-of-plane phonon modes $(0 \sim 6.25 \mathrm{THz})$. The largest EPC in graphene is about half the value of corner EPC in graphdiyne. 

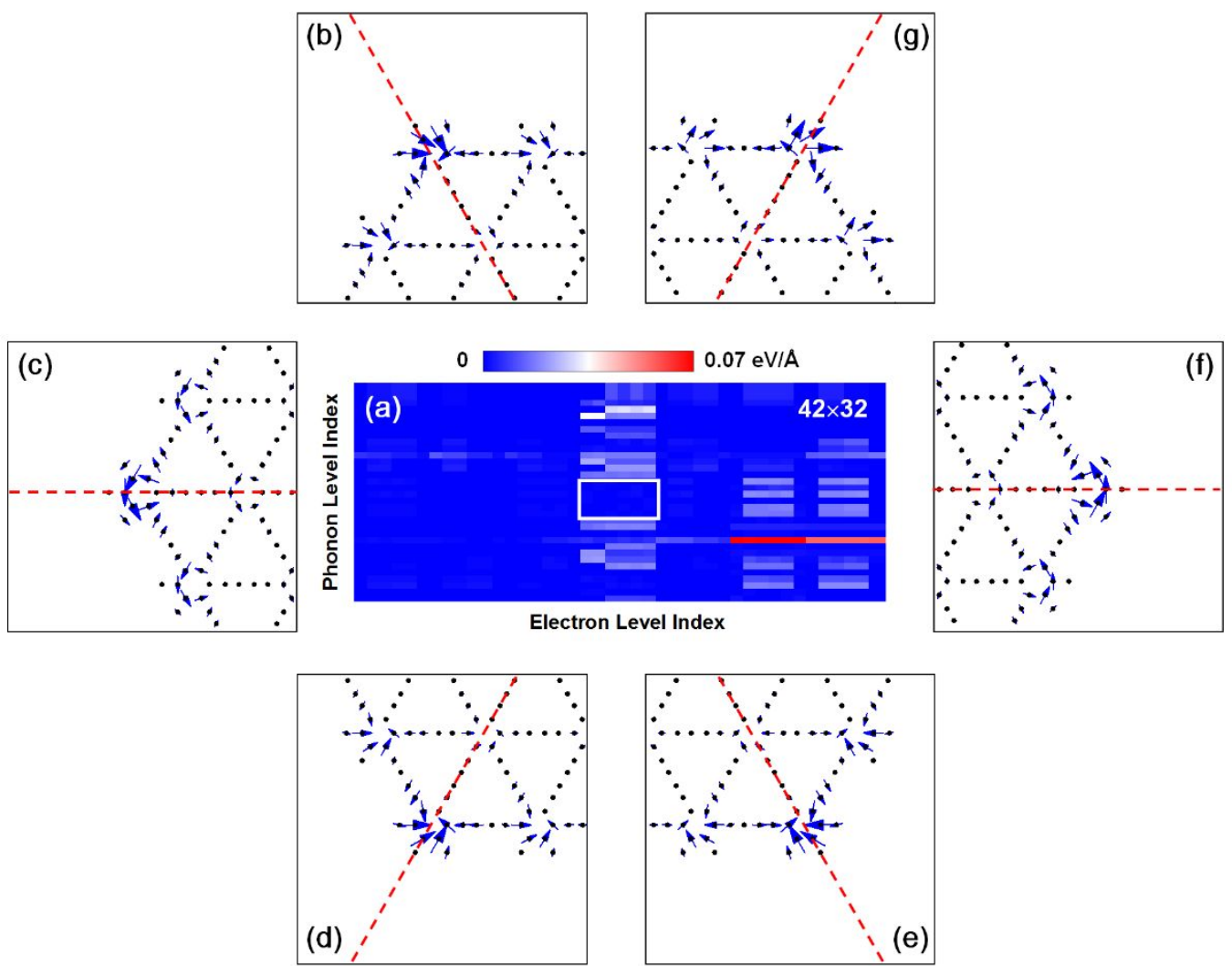

Figure S6. The EPC between 42 electron states around Fermi-level and 32 in-plane phonon modes around nontrivial gap (Fig. 2(f) in main text). The EPC between six phononic and electronic corner states is highlighted in the white rectangular region, showing no enhanced values. (b)-(g) Spatial distribution of six localized phononic corner states. The arrow direction and length denote the in-plane vibration direction and intensity of the phononic corner state. The red-dashed line denotes three in-plane mirror planes. 

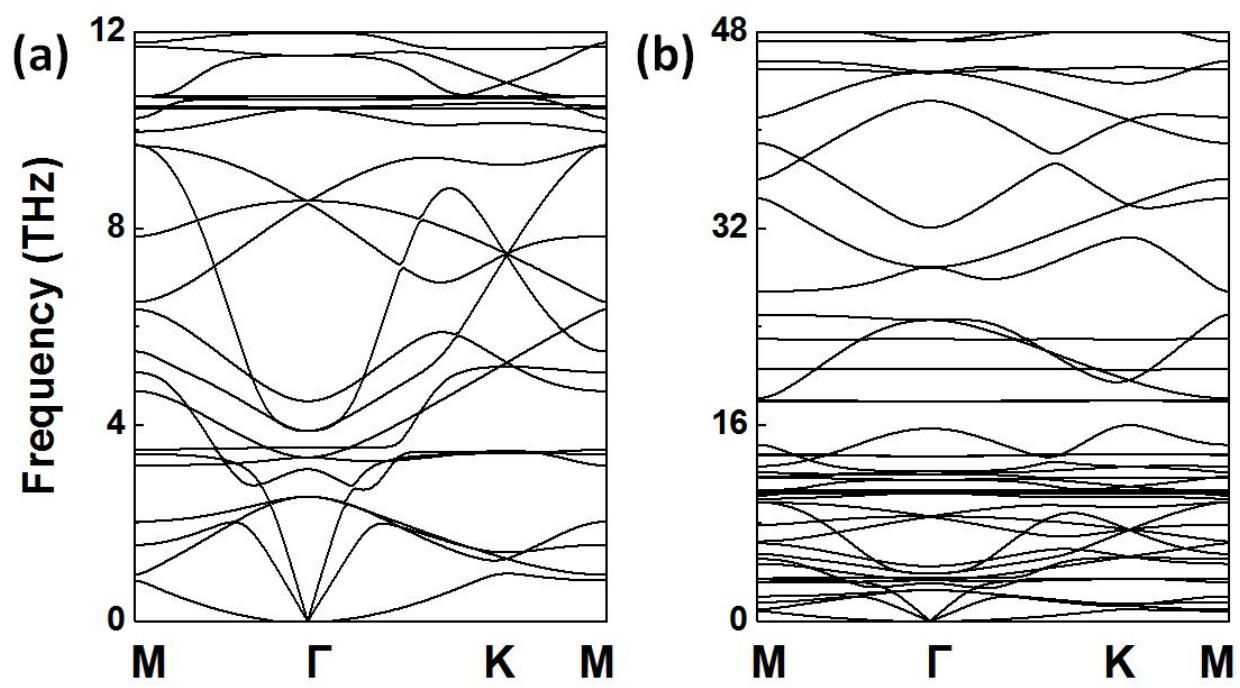

Figure S7. The full phonon bands of graphdiyne with both out-of-plane and in-plane contributions in smaller (a) and larger (b) frequency windows. 


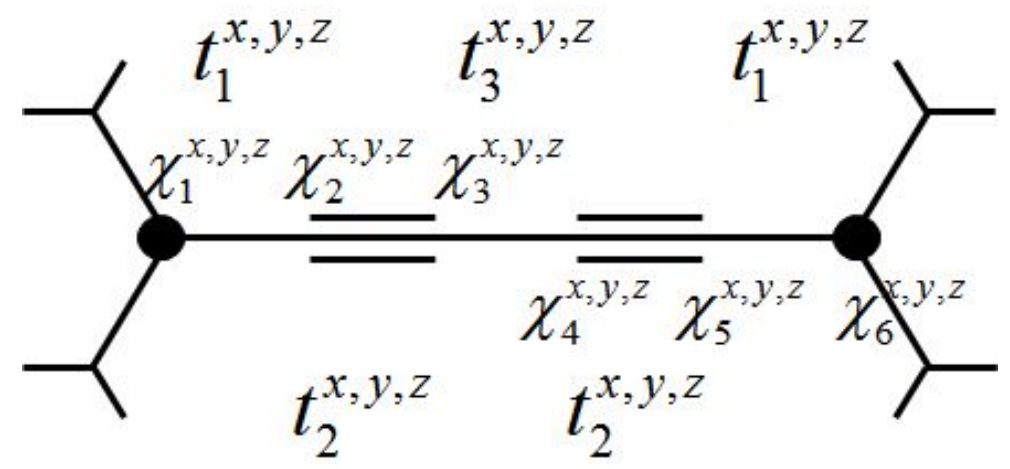

Figure S8. The phonon hopping between two vertex carbons (black dot) through three single-bonds (labeled with force constant $t_{1,3}^{x, y, z}$ ) and two triple-bonds (labeled with force constant $t_{2}^{x, y, z}$ ) in graphdiyne. The corresponding phonon modes at six carbons are labeled as $\chi_{1-6}^{x, y, z}$. 

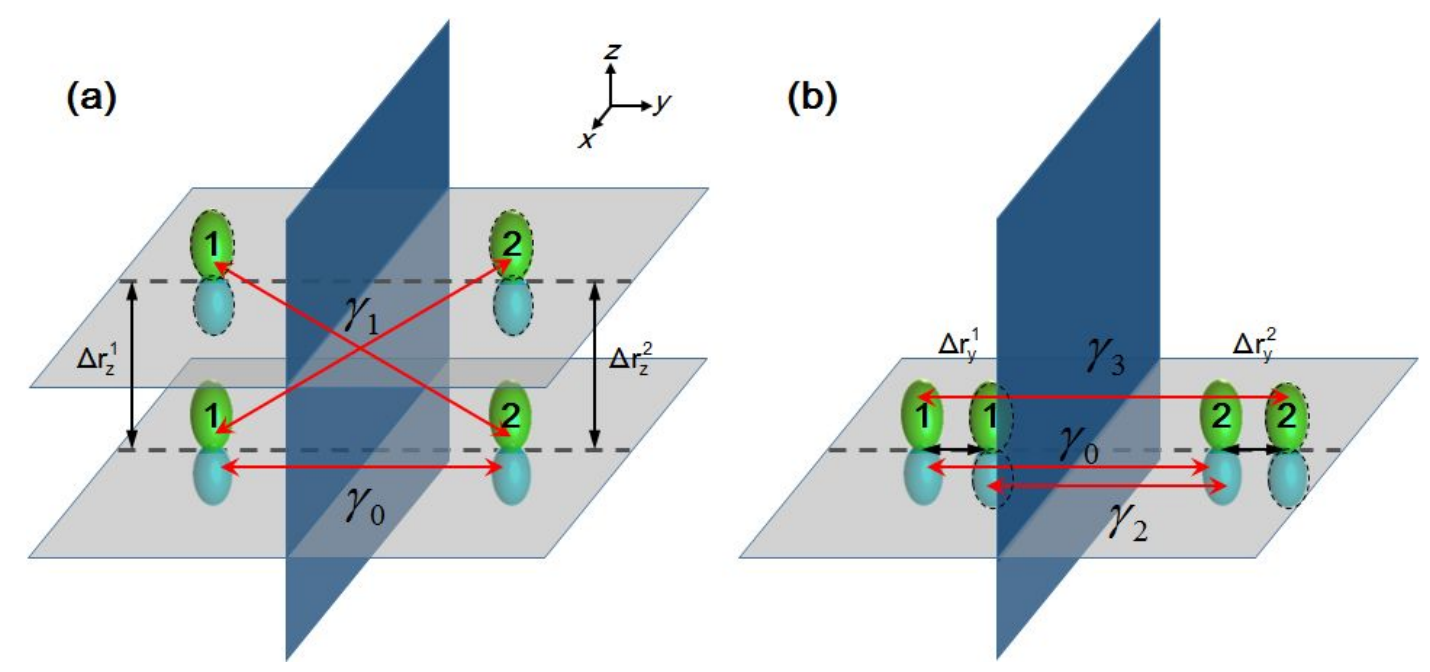

Figure S9. Schematic two-atom model with $p_{z}$ orbital, labeled as 1 and 2 . They are mirror symmetric to $x-z$ plane. (a) Out-of-plane phonon mode induced variation of electron hopping. If atom-1 (atom-2) has a tiny out-of-plane displacement of $\Delta r_{z}^{1}$ $\left(\Delta r_{z}^{2}\right)$, the electron hopping between them will change from $\gamma_{0}$ to $\gamma_{1}$. (b) In-plane phonon mode induced variation of electron hopping. If atom-1 (atom-2) has a tiny in-plane displacement of $\Delta r_{y}^{1}\left(\Delta r_{y}^{2}\right)$, the electron hopping between them will change from $\gamma_{0}$ to $\gamma_{2}\left(\gamma_{3}\right)$. The atom position after tiny displacement is highlighted by the dashed lines. The tiny displacement $\Delta r_{z}^{1}=\Delta r_{z}^{2}$ and $\Delta r_{y}^{1}=\Delta r_{y}^{2}$. 